\title{
DEVELOPMENT OF INTELLIGENT VERIFICATION MANAGEMENT SYSTEM FOR PRESSURE TRANSMITTER BASED ON LABVIEW
}

\author{
Zhao Mingli, Wu Meng* \\ College of Information \& Control Engineering, Jilin Institute of Chemical Technology, Jilin 132022, China. \\ *Corresponding Author Email: wu_meng@126.com
}

This is an open access article distributed under the Creative Commons Attribution License, which permits unrestricted use, distribution, and reproduction in any medium, provided the original work is properly cited

\section{ARTICLE DETAILS}

Article History:

Received 26 June 2018

Accepted 2 July 2018

Available online 1 August 2018

\section{ABSTRACT}

Based on LabVIEW, and the hardware GE PACE6000 modular pressure controller, a new kind of pressure transmitter intelligent verification management system is developed. The system communicates with PACE6000 through SCPI standardized instrument programming language and uses software program to achieve high precision fixed point control. The system can use SQL database technology to save the verification record directly, and also to query, delete, modify and print verification records. The system has extremely high accuracy and working efficiency, which can verify 5 pressure instruments with the same specification model in each time. The development of management system makes record management more intelligent. The system has been put into use in a testing center.

\section{KEYWORDS}

Lab VIEW, SCPI, Intelligent Verification.

\section{INTRODUCTION}

A pressure transducer or transmitter is a device or instrument that converts pressure into usable output signals (mostly electrical signals). Besides developing new principles and using new materials to develop new sensors, regular calibration and management of the metering performance of the pressure sensors and transmitters used are required in order to ensure and improve the testing accuracy and stability of the pressure sensor, especially long-term stability and extensive work ability to adapt to the environment. The traditional pressure transmitter verification uses mostly manual recording to record the verification data after verification and to calculate. The verification equipment is in independent working condition [1]. At least 20 manual operations are required for each verification, resulting in an extremely low efficiency, and the verification records cannot be saved effectively, bringing inconvenience or malpractice in the future. Therefore, more perfect intelligent network management are needed in instrument verification.

\section{TOTAL SYSTEM DESIGN}

The intelligent verification management system of pressure transmitter is built on the LabVIEW platform and is based on a high-performance pressure controller, realizing high precision fixed point control through software program. It designs and develops automatic verification management of intelligent pressure instrument, combined with the communication protocol of the commonly used in intelligent instruments. The system can be divided into 2 subsystems, the verification subsystem and the records management subsystem. The verification subsystem mainly involves the communication problems of LabVIEW and pressure controller and digital multimeter, while the records management subsystem involves the LabVIEW SQL 2005 database and SQL database access technology and report generation technology based on Microsoft Office.

The system adopts PACE6000 as the automatic pressure calibrating device, and verification of the pressure transmitter is done with the equipment including PLC and GALISH KE2000 DMM. Computer can communicate with several PACE6000 controllers which have different measurement range through Ethernet Switch, and is able to verify 5 pressure instruments with the same specification model in each time [15]. Users can set the parameters, such as the basic information of the instruments, the accuracy grade, the stroke numbers, the verified point numbers, the sampling time, etc. The verification system control and communicates with PACE6000 through SCPI communication protocol. PACE6000 provides the standard gas source for the calibrated transmitter according to the set value, and the output signal of the transmitter $(4 \sim 20 \mathrm{~mA}, 1 \sim 5 \mathrm{~V})$ as the feedback. Then the GALICH DMM KE2000 collects the standard signal from the transmitters and sends it to the PC. PC then calculates the basic error and return difference, judging whether the pressure transmitters qualified or not automatically, outputting a verification report. Finally, the verification record is stored in the SQL database for future queries.

\section{DEVELOPMENT OF VERIFICATION SYSTEM SOFTWARE}

\subsection{PACE 6000 SCPI Protocol}

PACE6000 is a modular intelligent pressure controller with extremely high control stability. There are two control channels with different measurement ranges and four kinds communication interfaces: Ethernet, GPIB, RS232 and USB. When configuring the future extended communication function through RS232 and IEEE interface, it can communicate with various pressure verification instruments. The system uses TCP/IP protocol interfaces, LabVIEW software programming and the open-ended standard commands for program-controlled instrumentation (SCPI) to communicate with several PACE6000 controllers [6]. The port number for communication is 5025.SCPI is a standardized instrument programming language using signal - oriented measurement. The commonly used SCPI Commands of PACE 6000 are shown as Table 1. 
Table 1: Common SCPI Commands of PACE 6000

$\begin{array}{cc}\text { Command } & \text { Description } \\ \text { :UNIT1/2 KPA/MPA... } & \text { Set the control channel } 1 / 2 \text {, the unit is KPA/MPA... } \\ \text { :SOUR1/2 } 30.000000 & \text { The setting value of the control channel } 1 / 2 \text { is } 30.0 \\ \text { :OUTP1/2 0/1 } & \text { Pressure control on/off to the control channel } 1 / 2 \\ : \text { CAL1/2:PRES:ZERO:AUTO } 1 & \text { Open the communication valve, set } 0 \text { to the control channel } 1 / 2 \\ : \text { CAL:PRES:ZERO:AUTO? } & \text { Finish the zero setting to the control channel } 1 / 2 ? \\ \text { CAL:ZERO:STOP } & \text { Close the communication valve, finish the zero setting } \\ : \text { SENS1/2? } & \text { Read the current pressure of channel } 1 / 2 \\ \text { :SOUR1/2:RANGE? } & \text { Read the pressure range of channel } 1 / 2\end{array}$

\subsection{Data Acquisition}

In accordance with the verification regulation GGJ882-2004, the verification device should meet special accuracy demands as a whole. The DAQ device has to reach the precision of four and a half bits, which cannot be achieved by average DAQ cards. So our system adopts GALISH KE2000 DMM with the precision of six and a half bits as the DAQ device. The PC communicates with KE2000 by serial port communication. The communication program is shown in Figure1.

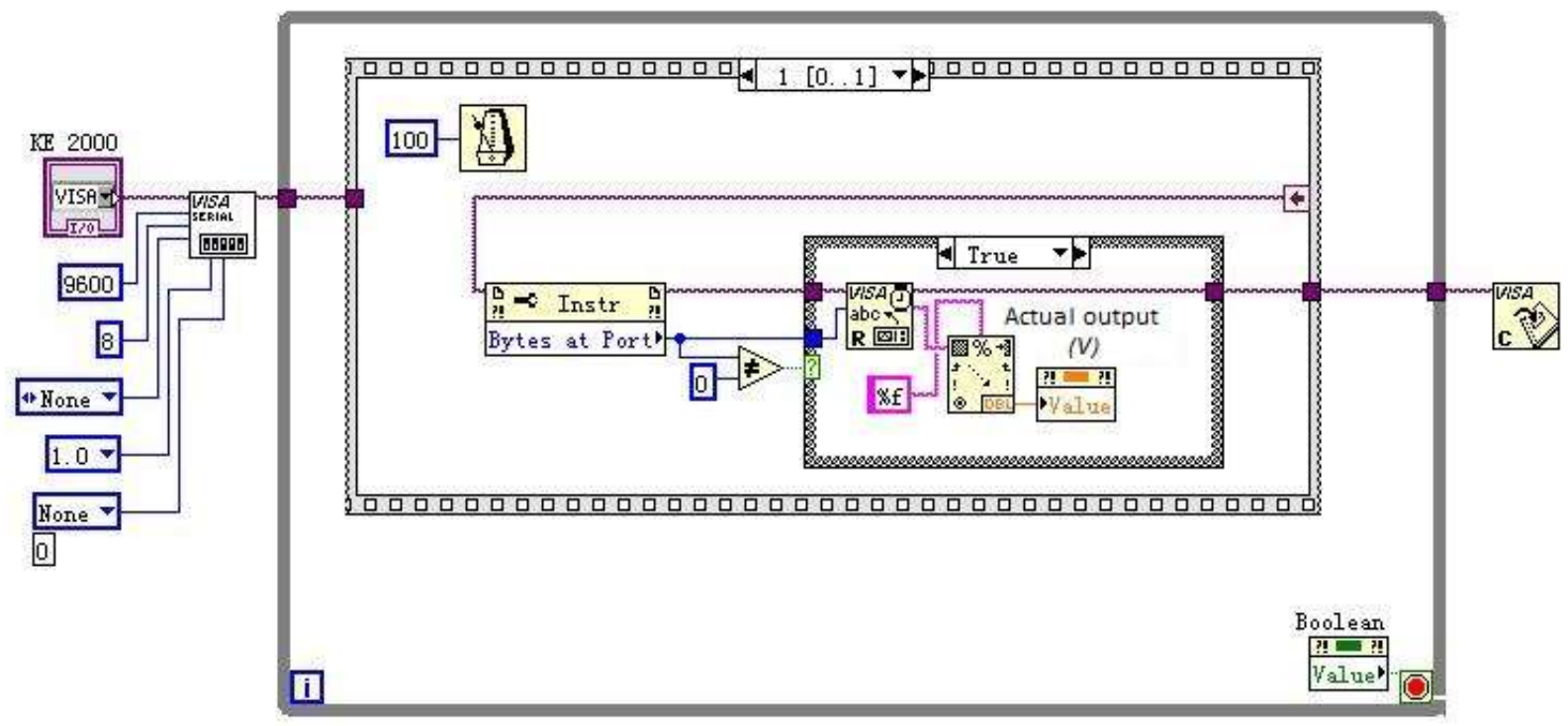

Figure 1: Communication Program between PC and KE2000

We use the standard input/output application programming interface VISA in LabVIEW to complete the instrument control. VISA uses the same functions and similar methods to control various instruments, including GPIB, serial port, USB, network, PXI, and VXI, etc. The difference is only in the configuration of the parameters. The serial communication function is located in the "instrument I/O - serial port" function, most of which are the VISA generic functions. It can also be found in the "instrument I/O - VISA" function. Functions as VISA configuration serial port, VISA writing, VISA read, VISA closing can be done.

\subsection{Multi-channel Signal Switch}

KE2000 DMM can only collect data of one channel in each time, consequently the multi-channel signals need to be switched in order to achieve the verification of more than one instrument at one time. The system uses LabVIEW as the upper computer and achieves the selection and switch of multi-channel signals by communicating with S7-200 PLC over PPI protocol. S7-200 is connected with several transmitters by Relays and selects multi-channel signals, implementing the time-sharing collecting of multi-channel analog signals [2].

The S7-200 communication physical interface is the RS-485 interface [3] PC needs an RS232-RS485 converter to communicate with S7-200. S7-200 does not require any programming, and all operations are done in LabVIEW. When the switching the signals, LabVIEW can complete the switch by simply writing the output state of q0.0-q0.4. The communication configuration interface of S7-200 PPI is shown in Figure 2.

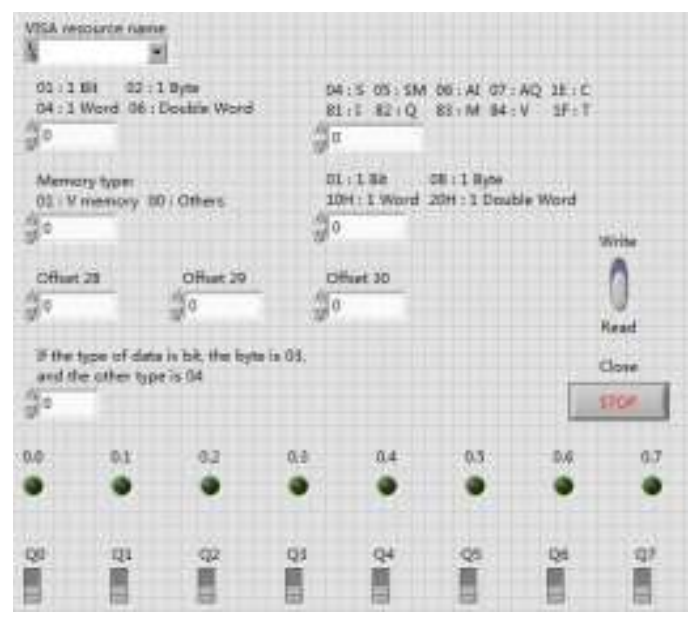

Figure 2: Communication Configuration Interface of S7-200 PPI. 


\section{DEVELOPMENT OF RECORDS MANAGEMENT SYSTEM SOFTWARE}

The record management system is based on the verification system. After completing the verification of the pressure instrument, the data is saved directly in the SQL database. We develop the management system, Retrieving the key words such as verification date, record number, manufacturer and factory number, then record and print the certificate [4].

The connection between LabVIEW and the SQL database is done through the database connection toolset. The system uses ODBC to connect with
SQL database. The process of ODBC access Database is Labview application - DCT function - ODBC API - ODBC driver - Database.

The connectivity process is as following: Firstly, build a database in SQL 2005 and name it "pace600.mdf"; Secondly, Build a form named "labviewdata" in the database; Thirdly, Open the ODBC data source manager to create a new SQL data source, and connect it to the SQL server then alter the default database to "pace600"; Then, Build a UDL file to connect with "pace6000"; Finally, Use the DB Tools Open Connection VI in LabVIEW to implement the connectivity [5]. The function that accesses the Database is in Connectivity-Database, and LabVIEW integrates many basic SQL statement functions that users can use directly. The functions and calling formats of the relational SQL commands are listed in Table 2.

Table 2: LabVIEW Accesses the SQL Database

\begin{tabular}{|c|c|}
\hline Function & SQL Command Format \\
\hline $\begin{array}{l}\text { Query by the verification date; (The results are ordered by the } \\
\text { record number.) }\end{array}$ & $\begin{array}{l}\text { select* from labviewdata } \\
\text { where VERIFICATION-DATE between } \\
\text { 'Y-M-D' and 'Y-M-D' } \\
\text { order by RECORD-NUMBER }\end{array}$ \\
\hline Query by the record number & $\begin{array}{l}\text { select * from labviewdata } \\
\text { where } R E C O R D-N U M B E R=' R E C O R D-N U M B E R^{\prime \prime}\end{array}$ \\
\hline Query by the factory number & $\begin{array}{l}\text { select* from labviewdata } \\
\text { where FACTORY-NAME='FACTORY-NAME' }\end{array}$ \\
\hline Query by the serial number & $\begin{array}{l}\text { select* from labviewdata } \\
\text { where SERIAL-NUMBER='SERIAL-NUMBER' }\end{array}$ \\
\hline Create a new record & insert into labviewdata (field1,field2...) values(value1,value2 ...) \\
\hline Update a record & $\begin{array}{l}\text { update labviewdata set field } 1=\text { value } 1 \\
\text { where } S E R I A L-N U M B E R={ }^{\prime} S E R I A L-N U M B E R^{\prime}\end{array}$ \\
\hline Delete a record & $\begin{array}{l}\text { delete from labviewdata } \\
\text { where SERIAL-NUMBER='SERIAL-NUMBER' }\end{array}$ \\
\hline
\end{tabular}

After all the verification work, the system need to generate and print the report. LabVIEW has office report generating tools itself, and after the edition 1.1.2, the toolkit provides Express VI-MS Office Report, which is based on interactive configuration. This Express VI can implement report generation conveniently [6]. The specific process is that, create a report in
Word, then bookmark and name a location in it. So in LabVIEW, the office report generating tools can determine the storage location by this bookmark, and after the report generation, print the report using the Print Report VI. The LabVIEW report printing program is shown in Figure3.

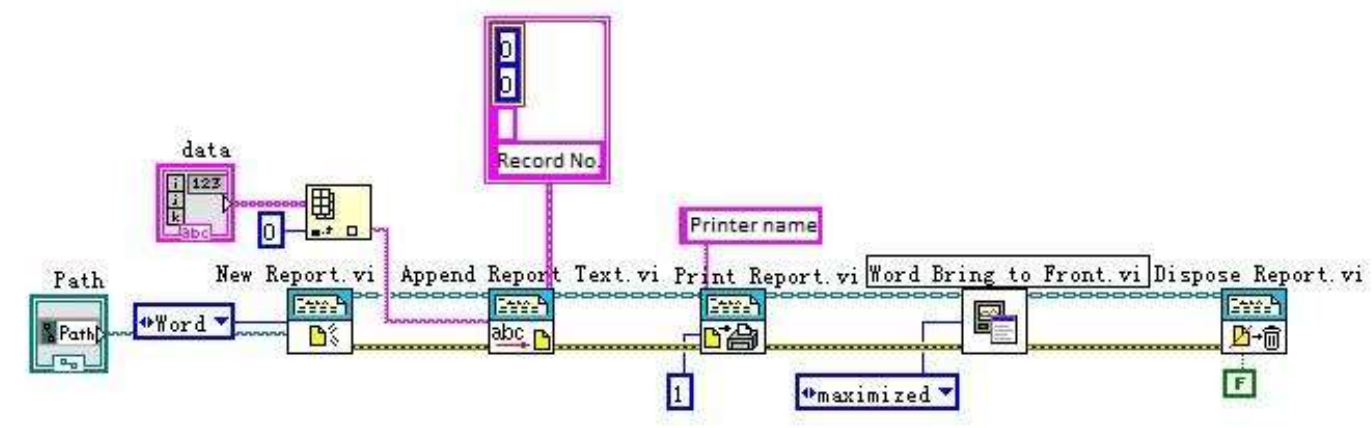

Figure 3: LabVIEW Report Rrogram

\section{CONCLUSION}

Through the second development of the verification system, the management system improves the traditional industrial pressure instrument verification and calibration mode by using LabVIEW software, and improves the management function of the verification system. It realizes the perfect combination of computer and standard instrument verification equipment. The system has perfect function and has been put into the actual instrument verification work. The system is proved to be efficient, reliable and has high intelligent management level by practice.

\section{REFERENCES}

[1] Kuanlin, F. 2007. Current Status and Outside of the Products of Pressure Transmitters in China Process. Automation Instrumentation, 28, 92-93.

[2] Dawei, H. 2013. Design and Implementation of Communication between LabVIEW and S7-200 PLC by PPI Protocol. Automation and Instrumentation, 04, 94-95+99+225.

[3] Zhongkai, C., Mengling, W., Lianyong, Z. 2011. The study of data communication based on S7-200 PLC. Industrial Instrumentation and
Automation, $04,78-80+89$.

[4] Xin, W. 2007. Design of Pressure Transmitter Management System. Shanxi Electric Power, 35 (4), 54-56.

[5] Guiming, Z., Zhiliang, Z. 2008. Accessing the database flexibly in Lab VIEW. Electronic Test, 09, 49-52+60.

[6] Huiqun, D., Fuming, W. 2011. Data communications based on the USB port in LabVIEW Electronic Test, 12, 37-40+61.

\section{AUTHOR INTRODUCTION}

Zhao Mingli (1967 -), Female, Han Ethnic Group, Meihekou city, JiLin Province, university degree, associate professor of college of information and control engineering of Jilin Institute of Chemical Technology, majoring in measurement and control technology and instrument automation.

Meng Wu (1974 -), Male, Jilin City, Jilin Province, Ph.D. in Jilin University, Changchun, China, 2009. Associate professor of college of information and control engineering of Jilin Institute of Chemical Technology. Mainly engaged in electromechanical transmission and automation research. 